\title{
ДОСЛІДЖЕННЯ ЕНЕРГЕТИЧНОГО СТАТУСУ ЛЮДИНИ ЯК ВАЖЛИВИЙ ЕТАП ПЕРЕХОДУ ДО 4П-МЕДИЦИНИ. ЧАСТИНА І: КОНЦЕПТУАЛЬНИЙ АНАЛІТИЧНИЙ ОГЛЯД
}

\author{
О. П. Мінцер, М. М. Потяженко ${ }^{1}$, Г. В. Невойт ${ }^{1}$ \\ Національна медична академія післядипломної освіти імені П. Л. Шупика \\ ${ }^{1}$ Українська медична стоматологічна академія
}

\begin{abstract}
Подальший прогрес медицини та вирішення проблеми неінфекційних захворювань можуть бути обумовлені онтологічним переосмисленням і глобалізацією сучасних наукових знань із позицій системної медицини. Тому постала необхідність на підставі онтологічного узагальнення наукових фрізико-біологічних уявлень сучасності з позицій системної медицини концептуально обґрунтувати поняття «енергетичний статус організму» та можливі методи його оцінювання. Авторами побудовано логічний ланцюг обґрунтування поняття, описано і пояснено онтологічні та методологічні аргументи методик можливого обстеження. Результати можуть сприяти досягненню вимог моделі 4П-медицини.
\end{abstract}

Ключові слова: онтологія, енергетичний статус, астенічний синдром, неінфекційні захворювання.

\section{THE STUDY OF THE ENERGY STATUS OF A PERSON AS AN IMPORTANT STAGE IN THE TRANSITION TO 4P-MEDICINE. PART I: CONCEPTUAL ANALYTICAL REVIEW}

\author{
O. P. Mintser, M. M. Potiazhenko ${ }^{1}$, G. V. Nevoit ${ }^{1}$ \\ Shupyk National Medical Academy of Postgraduate Education \\ ${ }^{1}$ Ukrainian Medical Stomatological Academy
}

\begin{abstract}
Background. Further progress in medicine and the solution of the problem of NCDs may be associated with an ontological rethinking and with the globalization of modern scientific knowledge from the perspective of systemic medicine. The purpose of the work is a conceptual substantiation of the concept of «energy status of an organism» and possible methods for its assessment on the basis of an ontological generalization of scientific physical and biological representations of the present from the standpoint of systemic medicine.

Materials and methods. The analysis of literature data was carried out in the course of conducting a search study of methods for express assessment of valeological status. This is a fragment of the initiative research work «Development of algorithms and technology for introducing a healthy lifestyle in patients with NCDs based on the study of psycho-emotional status» (state registration No. 0116U007798).

Results. The logical chain of substantiation of the concept is constructed by the authors in the article. Ontological and methodological factors of the methods of possible examination are described and explained by the authors in the work as well. The results can help achieve the requirements of the 4P medicine model.

Conclusions. The authors propose for the first time: 1) the introduction of the concept of the patient's energy status; 2) a shift in the focus of clinical attention to the indicator of muscle content in the body, as another new, early predictor of the occurrence of NCDs and metabolic disorders of the body; 3) the use in practical health care of a personified method for determining the direction of correction of a patient's life style, the formula of which provides for the implementation of a complex of instrumental diagnostics: instrumental bioimpendanceometry, spectral analysis of heart rate variability and variational pulsometry, skin galvanic rapid testing, EPI method with subsequent analysis of the results from physiological position assessment of the energy status of the body; 4) an ontological model of the etiopathogenesis of CFS as a state of energy deficiency of the body due to the failure of the physiological adaptation mechanism at the level of central regulatory mechanisms of hypothalamic structures and methods of possible instrumental verification of CFS.
\end{abstract}

Key words: ontology, Energy Status, Chronic Fatigue Syndrome, Noncommunicable Diseases. 


\title{
ИССЛЕДОВАНИЕ ЭНЕРГЕТИЧЕСКОГО СТАТУСА ЧЕЛОВЕКА КАК ВАЖНЫЙ ЭТАП ПЕРЕХОДА К 4П-МЕДИЦИНЕ. ЧАСТЬ І: КОНЦЕПТУАЛЬНЫЙ АНАЛИТИЧЕСКИЙ ОБЗОР
}

\author{
О. П. Минцер, М. М. Потяженко ${ }^{1}$, А. В. Невойт ${ }^{1}$ \\ Национальная медицинская академия последипломного образования имени П. Л. Шупика \\ ${ }^{1}$ Украинская медицинская стоматологическая академия
}

\begin{abstract}
Дальнейший прогресс медицины и решение проблемы неинфекционных заболеваний могут быть обусловлены онтологическим переосмыслением и глобализацией современных научных знаний с позиций системной медицины. Поэтому появилась необходимость на основании онтологического обобщения научных фризико-биологические представлений современности с позиций системной медицины концептуально обосновать понятие «энергетический статус организма» и возможные методы его оценки. Авторами построена логическая цепочка обоснования понятия, описаны и объяснены онтологические и методологические фракторы методик возможного обследования. Результаты могут способствовать достижению требований модели 4П-медицины.
\end{abstract}

Ключевые слова: онтология, энергетический статус, астенический синдром, неиноекционные заболевания. 
Введение. Необходимо отметить, что сегодня накоплен значительный пласт принципиально нового для медицины научного знания относительно сущности протекания электромагнитных феноменов в живых биологических системах и в человеческом организме, как в частном их проявлении. Открыты и изучены электрические, магнитные параметры, частотные характеристики отдельных клеток, тканей, органов и организма в целом. Доказаны факты порождения живыми клетками переменных электромагнитных полей [1, 10-12, 27], наличия у всех биологических образцов, в том числе и у человеческого тела, феномена сверхслабой эмиссии фотонов (в англоязычной литературе — Ultra-Weak Photon Emission (UPE) [17, 30, 32, 36]), переноса электронов вдоль пептидных цепей белковых молекул удлиненной волной деполяризации - солитоном [4, 10, 27], существования нового анатомического образования, названного первичной сосудистой системой (в англоязычной литературе Primo Vascular System (PVS) [6, 15, 24, 27, 31, 34]), открыт новый путь трансформации механической энергии в электрическую - пьезобиосинтез [1, 2, 10, 27]. Углубилось трансдисциплинарное понимание зависимости протекания электромагнитных процессов и энергетического обмена в системе «ближний космос — планета Земля — биоценоз — человек» $[11,12]$. Продолжают изучаться ряд частных и фундаментальных закономерностей фолдинга протеинов, репликации нуклеиновых кислот, репарации и биосинтеза тканей и т.д. На сегодняшний день уже общепризнано, что фотон, как фундаментальная единица электромагнитного поля, играет важную роль в функционировании человеческого организма [1, 8, 10-12]. Результатами системного трансдисциплинарного подхода являются возможности оценки электромагнитных феноменов в человеческом организме и достигнутые успехи магнитографии, компьютерной томографии и магнитоядерного резонанса. Однако в медицине вопросы электромагнитных характеристик органов освещены незначительно, исключительно в рамках специальностей их изучения и узких научных направлениях исследований. В тоже время, для дальнейшего прогресса практического здравоохранения и решения такой актуальной проблемы как пандемия неинфекционных заболеваний (НИЗ) на современном этапе необходимы систематизация, онтологическое переосмысление всех принципиально новых трансдисциплинарных знаний касательно организации вещества и его функционирования в живой материи, включая человеческий организм, с созданием универсума научного знания. Глобализация научных знаний и их анализ с позиций системной медицины будут способствовать формированию более совершенных методологий познания, а благодаря значительному развитию компьютеризированных технологий - созданию и внедрению в практическое здравоохранение принципиально иных, новых, альтернативных диагностических и лечебных подходов.

Цель работы: на основании онтологического обобщения научных физико-биологических представлений современности с позиций системной медицины концептуально обосновать понятие «энергетический статус организма» и возможные методы его оценки.

Материалы и методы исследования. Онтологический анализ данных выполнялся в ходе поискового исследования методов экспрессоценки валеологического статуса как фрагмент инициативной научно-исследовательской работы «Разработка алгоритмов и технологии внедрения здорового способа жизни у больных неинфекционными заболеваниями на основании изучения психоэмоционального статуса» (номер госрегистрации 0116U007798).

Результаты и их обсуждение. Обоснование логики методологии оценки энергетического статуса. В ходе выполнения научного поиска методик экспресс-диагностики валеологического статуса было отмечено, что функциональное состояние человеческого организма является недостаточно исследованным компартментом с позиций доказательной медицины. С целью большей наглядности для изучения нами была взята в качестве отправной точки онтологического исследования клинически признанная форма нарушения функционального состояния - астенический синдром (в англоязычной литературе - Chronic Fatigue Syndrome (CFS)). В международной классификации болезней 10-го пересмотра состояния CFS рассматривается в рубриках «Неврастения F48.0», «Органическое эмоционально-лабильное (астеническое) расстройство F06.6», «Синдром усталости после вирусной инфекции G93.3», другие астенические расстройства (неспецифическая астения R53; синдром выгорания - Z73.0; поствирусный астенический синдром - G93.3; психастения - F48.8), в том числе и в педиатрической практике (синдром утомляемости после перенесенной 
вирусной болезни - G93.3; энцефалопатия неуточненная - G93.4; задержка этапов развития - R62.0, реакции на тяжелый стресс и нарушения адаптации - F43, F43.0-F43.2, F43.20-F43.25, F43.28, F43.8; умственная отсталость неуточненная - F79; специфические расстройства развития речи и языка - F80, F80.0-F80.2; специфические расстройства развития учебных навыков - F81, F81.0-F81.3, F81.8, F81.9; специфические расстройства развития моторной функции - F82; смешанные специфические расстройства психологического (психического) развития - F83; общие расстройства психологического развития — F84, F84.8, F84.9) и т.д. Примечательно, что на сегодняшний день CFS остается едва ли не единственным абсолютно объективно не диагностируемой с позиций доказательной медицины патологией - специфическая лабораторная и инструментальная диагностика CFS отсутствует, не смотря на значительный пласт проведенных научных исследований. Установление CFS основывается исключительно на соответствующих жалобах пациента, а его существование, не смотря на отсутствие объективного подтверждения, не подвергается сомнению, хотя бы по той причине, что каждый человек испытывал подобные ощущения лично и у научного сообщества нет сомнений на этот счет. CFS изучается как синдром практически при всех физических, психосоматических, психических заболеваниях. Механизм CFS также остается не понятым до конца ни одной специализированной отраслью медицины в традиционном редукционном подходе [7, 9, 20].

В ходе выполняемого исследования онтологии энергетического обмена и анализа общеизвестных научных данных с позиции системной медицины нами была построена следующая логическая цепочка выводов в аспекте моделирования решения этого вопроса:

(1) исходя из системных онтологических позиций и из понятия, что человеческий организм является сложной саморегулирующейся системой, CFS - это универсальный психофизиологический механизм сохранения жизнедеятельности системы путем сохранения энергии посредством отказа и прекращения как физической, так и умственной деятельности (в разной мере) в ответ на любое состояние, угрожающее истощением энергетических ресурсов;

(2) истощение энергетических ресурсов может развиваться вследствие комплексного действия экзогенных (психосоциальных, инфекционных, бионических, антропогенных и т.д.) и эндогенных (инфекционно-иммунных, метаболических, нейрогуморальных) факторов; наиболее значимой теорией считается вирусная, поскольку вирусы, персистируя в центральной нервной системе, могут служить триггерными факторами;

(3) ключевыми в процессе возникновения CFS у человека являются изменения деятельности центральной нервной системы, проявляющиеся активацией систем лимбико-ретикулярного комплекса, обуславливающего адаптивное поведение в ответ на любые виды стресса, а именно: изменения функционирования ретикулярной формации ствола мозга (обеспечивает поддержание уровня внимания, восприятия, бодрствования и сна, общую и мышечную активность, вегетативную регуляцию), гипоталамо-гипофизарно-адреналовой системы (обеспечивает ключевую нейрогормональную регуляцию ответа на стресс), а также иммуннометаболические (уменьшение мозгового нейротрофического фактора, активация системы цитокинов мозга, повышение лактата в церебральной жидкости, в серотонинергической нейромедиаторной системе и т.д.) и макроскопические изменения (локальные снижение мозгового кровотока, состояния белого и серого веществ);

(4) изменения функционирования центральной регуляции передаются через гипатоламо-гипофизарно-надпочечниковую ось на эндокринные железы, обуславливающие изменения метаболизма на тканевом уровне и через изменения активности звеньев вегетативной нервной системы (симпатического и парасимпатического отделов);

(5) в норме работа вегетативных отделов нервной системы сбалансирована; (как правило) стрессовые реакции сопровождаются активацией симпатической нервной системы с последующим ее истощением и возникновением относительной парасимпатикотонии; последующее перенапряжение парасимпатического отдела ведет к его истощению и развитию стойкой симпатикотонии (упрощенная теза);

(6) регуляция сердечно-сосудистой деятельности осуществляется посредством центрального (головной мозг и гуморальная регуляция) и автономного (симпатический и парасимпатический отделы вегетативной нервной системы) контуров - соответственно параметры сердечной деятельности отображают активность этих регуляторных механизмов и могут быть зарегистрированы методами 
магнитокардиографии, вариабельности ритма сердца (ВРС);

(7) ток крови в соответствии с пульсовыми волнами сердечной деятельности, которые зависят от выше обозначенных мио- и нейрогенных механизмов регуляции, передает динамику сокращений на периферические ткани микроциркуляторного русла, феномен «мерцания капилляров» дозирует массоперенос вещества из крови в интерстиций и обратно в сосудистое русло, лимитирует кинетику окислительно-восстановительных (энергетических) процессов в тканях — эти параметры могут быть зарегистрированы такими рутинными методами как реовазография, реоэнцефалография, плетизмография и перспективными - кожно-гальваническое тестирование, газоразрядная визуализация/регистрация эмиссии фотонов в газовом разряде, фоторегистрация эмиссии фотонов и т.п.;

(8) конечное звено - нарушение тканевого/ мышечного метаболизма, проявляющееся переключением процессов энергообразования на менее эффективный анаэробный путь, что способствует патологическому нарастанию уровня молочной кислоты даже после небольшого физического напряжения и клинически проявляется усталостью, слабостью, болью и мышечными спазмами; возникает оксидативный стресс, приводящий к повреждению наиболее важных полимеров нуклеиновых кислот, белков и липидов клеточных мембран, полиненасыщенных жирных кислот, инактивации эндогенного оксида азота (NO) с образованием токсичных пероксинитритов, что приводит к иммунным нарушениям (снижение IgG за счет прежде всего G1- и G3-классов, числа лимфоцитов с фенотипом CD3 и CD4, естественных киллеров, повышение уровня циркулирующих комплексов и антивирусных антител разного типа, повышение $\beta$-эндорфина, интерлейкина-1 и интерферона, а также фактора некроза опухолей и т.д.); возникающий дисбаланс в системе цитокиновой сети интерлейкинов способствуют возникновению местного и системного повреждающего действия; для головного мозга дисбаланс уровней цитокинов является сигналом болезни, требующими изменениями энергетических приоритетов организма и активации центрального звена регуляции - систем лимбико-ретикулярного комплекса и т.д. - что и замыкает порочный круг этиопатогенеза.

Анализируя полученную онтологическую модель этиопатогенеза астенического синдрома приходим к заключению, что результаты применения ряда инструментальных методик могут нам позволить объективно оценивать функциональную реакцию организма на действие факторов и разработать методологию объективной диагностики как CFS, так функционального состояния организма в целом для применения в практическом здравоохранении, а также для контроля и прогнозирования реакций пациента в ходе лечения согласно модели 4П-медицины.

Онтологическое обоснование понятия «энергетический статус». В ходе выполнения научного поиска методик экспресс-диагностики валеологического статуса и онтологического анализа общеизвестных научных данных было отмечено, что на сегодняшний день существуют ряд сертифицированных методов оценки функционального состояния человека. Как наиболее релевантный метод для скрининговой инструментальной оценки энергетического статуса пациента для возможного использования в практическом здравоохранении нами был определен спектральный анализ ВРС. Использование показателей спектрального анализа повышает клиническую валидность метода, делая его значимым для практического здравоохранения. Спектральные компоненты ВРС имеют доказанные физиологические корреляты, являются маркерами вегетативной активности и соответственно отображают характер и интенсивность регуляторного воздействия на энергетику организма: влияние головного мозга и гипатоламо-гипофизарно-надпочечниковой оси (центральный контур) проявляется в виде низкочастотного диапазона волн (25-66 с, 0,04-0,015 Гц) - Very Low Frequency (VLF), симпатического отдела — низкочастотными волнами (6,6-25 с, 0,15-0,04 Гц) — Low Frequency (LF), парасимпатического - волнами высокочастотного диапазона (2,5-6,6 с, 0,4-0,15 Гц) — High Frequency (HF). Соответствующее доминирование типа волн указывает на тип основной регуляции, при этом у здорового человека выражены и сбалансированы все три типа волн, на начальных стадиях патологии могут преобладать HF (парасимпатика) или LF (симпатика), при выраженной патологии, когда имеет место истощение автономного контура, доминирует VLF (центральный контур), у лиц пожилого и старческого возраста, тяжело больных, в состоянии приближения естественной смерти наблюдается низкий уровень регуляции обоих контуров и формирование ригидного ритма 
сердечной деятельности, при котором регуляция имеет минимальную выраженность. Оценка диапазонов и их пиков имеет значительную клиническую ценность при сопоставлении с катамнезом пациента с целью объективного прогнозирования и оценки эффективности терапии НИЗ. Важными показателями энергетического статуса являются вегетативный баланс — LF/HF (симпатика/ парасимпатика) и общая мощность спектра сердечной деятельности — Total power (TP). Если LF/HF составляет величину выше единицы, то у пациента преобладает процесс расхода энергии, если ниже единицы - преобладает процесс восстановления энергии. Сравнение ТР с показателями нормы (1500-5000 мс²), установленными Европейским Кардиологическим Обществом и Северо-Американским Обществом Стимуляции и Электрофизиологии (1996), дает возможность оценить суммарный энергетический потенциал, который снижается при патологии и существенно снижается при тяжелой патологии и приближении естественной смерти [5].

Проведенные нами исследования энергетического статуса пациентов на базе центра биофотоники и валеологии Украинской медицинской стоматологической академии подтвердили данные литературы, что показатели сердечной деятельности достоверно различаются в группах функционально здоровых молодых людей с различной степенью физической тренированности, с достоверным снижением $(\mathrm{p}<0,0001)$ спектра у нетренированных $(\mathrm{n}=23$,

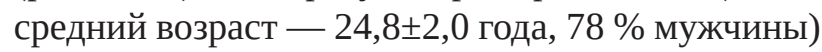
в сравнении с профессиональными спортсменами $(\mathrm{n}=60$, средний возраст - 20,5 \pm 4 года, $100 \%$ мужчины): $\mathrm{TP}=2918 \pm 2042$ против $24173 \pm 71872$ при норме 3466 \pm 1018 мс $^{2}, \mathrm{VLF}=935,5 \pm 858,2$

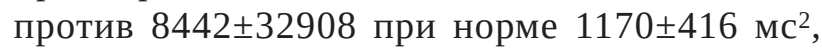
$\mathrm{LF}=1080 \pm 808,7$ против 7369 \pm 29211 при норме $1170 \pm 416$ мс $^{2}, \mathrm{HF}=902,4 \pm 759,2$ против 8362 \pm 14530 при норме $975 \pm 203$ мс $^{2}$. С учетом того, что данные показатели являются результатом, отображающим регуляторное метаболически-энергетическое воздействие центрального звена - гипоталамо-гипофизарно-надпочечниковой оси, нами предлагается рассматривать их как энергетические и использовать как составляющие оценки энергетического статуса в комплексной диагностике.

Полученные результаты энергетических показателей мощности сердечной деятельности коррелировали с результатами состава тела обследованных лиц. Показатели были достоверно выше у лиц, систематически занимающихся спортом и имеющие достаточное (согласно возрастной норме) содержание мышц в организме. У нетренированных лиц в сравнении со спортсменами был установлен дефицит мышечной массы 37,4+5,7 против

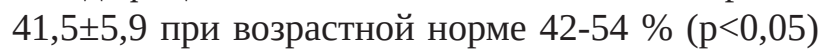
и нарушения жирового обмена - повышенное

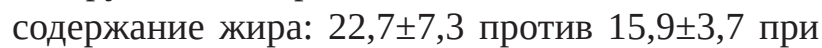
норме 8-19,9 \% (p<0,05); при персональном анализе у 11 (48 \%) респондентов было диагностировано предожирение, у 2 (8 \%) - висцеральное ожирение в сравнении с нормой состава тела у всех спортсменов. С позиций системной медицины мышцы отвечают не только за механическое передвижение, а и выполняют ключевую метаболическую роль, с одной стороны, являясь т.н. «периферическим сердцем», обеспечивая своим сокращением возврат крови к сердцу, с другой трансформируя универсальный носитель энергии аденозинтрифосфат в другие виды энергий (механическую, электрическую, электромагнитную), являясь «энергетической станцией» организма. При этом у людей, систематически занимающихся спортом данные процессы трансформации, а по сути — конечного усвоения энергии, протекают в наилучшем энергетическом режиме. Отсутствие систематических физических нагрузок, как еще раз показало наше исследование, приводит к гипотрофии/дефициту мышц и как следствие сопряжено с более низкими энергетическими показателями сердечной деятельности и с нарушением утилизации энергии, а именно с избыточным ее накоплением в виде разных форм ожирения при условии несбалансированности питания. В этой связи нами предлагается использования инструментального показателя процентного содержания мышц в организме в качестве обязательного дополнительного критерия метаболического/энергетического статуса организма и как раннего предиктора возникновения НИЗ. Декларируемая нами необходимость смещения акцентов внимания клиницистов с жировой ткани на мышечную также способна логически объяснить существующий так называемый парадокс ожирения, когда многочисленные исследования подтверждают повышенный риск развития хронической сердечной недостаточности при ожирении $[19,28]$, при этом ряд исследований свидетельствуют, что возникновение ожирения уже при возникшей сердечной недостаточности наоборот улучшает прогноз этих пациентов [3, 26, 29]. Дело в том, что в значительном количестве 
данных исследований для диагностики ожирения был использован индекс массы тела, который на сегодняшний день уже нельзя считать в полной мере адекватным показателем. В этой связи в научной работе по оценке выживаемости больных с сердечной недостаточностью [3] была выполнена дополнительно антропометрия с измерением толщины кожно-жировой складки в четырех точках с помощью калипометра с расчётом процента жировой массы тела по формуле Durnin-Womersley (1991), измерение окружности плеча ненапряженной и напряженной руки, талии и бедра и установлено, что лучшая выживаемость имела место у пациентов с большей толщиной кожной складки над лопаткой и бицепсом. Данный феномен авторами был объяснен тем, что пациенты с хронической сердечной недостаточностью и ожирением имеют «метаболический резерв» в виде жировой ткани относительно больных, имеющих признаки кахексии. Мы ставим под определенное сомнение этот вывод авторов, поскольку, исходя из предлагаемых нами позиций, очевидно, что данный показатель может быть объяснён за счет уровня наличия именно мышечной массы в области бицепса и т.п. Вышеизложенное также обуславливает целесообразность введения в процедуру обследования энергетического статуса пациентов инструментальной биоимпендансометрии, которая при соблюдении методики позволяет объективно сделать вывод о составе тела и метаболизме человека.

Перспективным сертифицированным методом измерения электромагнитных и энергетических характеристик человеческого организма является метод анализа эмиссии фотонов в газовом разряде (исторические прототипы - газоразрядная визуализация, кирлианография) из кожи пальцев человека (в английской литературе - Electron Photonic Imaging (EPI)). Сущность EPI метода, в том, что под действием короткого электрического импульса (10 микросекунд) генерируется отклик кожной ткани в виде возбужденной газовой плазмы (разряда), которая излучает свет (фотоны), регистрируемые прибором посредством цифровой камеры и передаваемые в компьютер для последующей обработки и анализа в виде графического файла. Диагностические возможности ЕРI метода продолжают совершенствоваться и связаны с улучшением программного обеспечения для анализа показателей и с оптимизацией технических характеристик оборудования [27] и как следствие валидности. Это позволяет рассматривать ЕРI метод как релевантный для индивидуальной оценки здоровья человека [16, 21, 22, 33, 37], в том числе в спортивной медицине [23, 35]. Нами в клиникоэкспериментальном исследовании использовался прибор Био-Велл 2.0, имеющий европейскую сертификацию измерительного прибора [18].

Предварительные результаты исследований показали существующую связь между сердечной деятельностью и показателями излучения на пальцах. Полученные результаты согласуются с работами [13], в которых освещены биофизические и физиологические механизмы микроциркуляции и регуляции сосудистого тонуса, связанные с процессом электрофотонной эмиссии с поверхности кожи в газовом разряде. Дальнейшее экспериментальное подтверждение гипотезы позволит дополнить методологию обследования энергетического статуса пациента показателями анализа EPI метода.

Энергетический статус и 4П-медицина. Сегодня концепция 4П медицины является одной из самых перспективных моделей здравоохранения, поскольку направлена не высокий уровень индивидуализации диагностических и лечебных подходов, исходя из четырех базовых принципов: прогнозирование, профилактика, персонализация, партисипация/задействование пациента в диагностический и лечебные процессы [14]. Для возможности введения данных подходов в существующую модель медицины важна разработка способов и инструментов для их реализации в практическом здравоохранении. В ходе решения этой задачи был взят за основу разработанный нами способ персонифицированного определения направления коррекции способа жизни пациента, формула которого предусматривает выполнения инструментальной импендансометрии, тестирования по опроснику состояния становления здорового образа жизни (ЗОЖ) и определения предикторов возникновения НИЗ с последующим автоматизированным расчетом с помощью программного обеспечения параметров частотного анализа ВРС и показателей вариационной пульсометрии. Выполнение методики занимает около 20 минут. Дополнительное применение в расширенной версии метода кожно-гальванического экспресс-тестирования и ЕРI метода могут удлинить процедуру на 5-20-30 минут в зависимости от алгоритма исследования. Принципиально важное значение имеет клиническая трактовка полученных показателей, которую предлагается производить исходя из физиологических позиций интерпретации энергетического 
статуса организма. Принципиальная новизна и практическая ценность подобного подхода заключается в возможности работы как с функционально здоровыми людьми для оценки уровня их здоровья, первичной профилактики НИЗ, так и с пациентами на различных стадиях лечения НИЗ, реализуя на практике принципы модели 4П-медицины.

Выводы. Таким образом, нами впервые предлагается: 1) введение понятия энергетического статуса пациента - как объективно существующего комплекса из результатов современных сертифицированных методов инструментальной диагностики на основании онтологического ресинтеза современных научных знаний; 2) смещение акцентов клинического внимания к показателю содержания мышц в организме, как к еще одному новому, раннему предиктору возникновения НИЗ и нарушения метаболизма организма; 3) использование в практическом здравоохранении способа персонифицированного определения направления

\section{Литература.}

1. Квантово-биологическая теория / В. В. Бойко, М. А. Красноголовец (ред.). — Харьков: Факт, 2003. 967 c.

2. Пьезобиосинтез:предпосылки, гипотезы, факты / В. В. Бойко, Е. И. Сокол, П. Н. Замятин (ред.). — Харьков: Харьк. политехн. ин-т, 2016. - Монография, B $4 \mathrm{~T}$.

3. Прогностичне значення втрати маси тіла та показників антропометрії у пацієнтів із хронічною серцевою недостатністю та зниженою фракцією викиду лівого шлуночка / К. В. Войцеховська, Л. Г. Воронков // Український медичний часопис. - 2019. - № 5 (2). - С. 31-34.

4. Давыдов А. С. Солитоны в молекулярных системах / А. С. Давыдов. - К.: Наукова думка, 1984. 288 c.

5. Дорошенко Л. Ю. Вариабельность сердечного ритма: забытое наследие космической медицины СССР]. — Режим доступа: http://vdd-pro.ru/ru/2020/01/hrvanalysis-forgotten-heritage/.

6. Сучасні погляди на субстрат та механізми дії акупунктури / О. Є. Коваленко, М. Є. Чіжикова // Міжнародний неврологічний журнал. — 2017. — № 6. - С. 120-126.

7. Астенический синдром в практике невролога и семейного врача / О. В. Котова, Е. С. Акарачкова // Русский медицинский журнал. - 2016. — № 13. С. 824-829. коррекции способа жизни пациента, формула которого предусматривает выполнения комплекса инструментальной диагностики: инструментальной биоимпендансометрии, спектрального анализа вариабельности сердечного ритма и вариационной пульсометрии, кожно-гальванического экспресстестирования, EPI метода с последующим анализом результатов с физиологических позиции оценки энергетического статуса организма; 4) онтологическая модель этиопатогенеза CFS как состояния энергетического дефицита организма в следствии срыва физиологического механизма адаптации на уровне центральных регуляторных механизмов гипоталамических структур и методы возможной инструментальной верификации CFS.

Результаты представленного концептуального обзора являются основой для проведения дальнейших клинико-экспериментальных исследований с целью улучшения диагностики и профилактики НИЗ путем разработки и внедрения в медицинскую практику современных наукоемких технологий.

8. Системна біомедицина (у двох томах). Т. 1. Концептуалізація / О. П. Мінцер, В. М. Заліський. - К.: Інтерсервіс , 2020. - 552 с.

9. Пизова Н. В. Клиника, диагностика и лечение синдрома хронической усталости / Н. В. Пизова // Медицинский совет. - 2015. - № 2. C. 20-24.

10. Потяженко М. М. Энергетическая система человека в свете современных физико-биологических знаний, концепций, гипотез / М. М. Потяженко, А. В. Невойт // Український медичний часопис. - 2019. — № 4 (132), T. 2. - С. 24-29.

11. Энергетическая система человека как часть природного энергообмена: значение для фундаментальной науки и медицины (часть I) / М. М. Потяженко, А. В. Невойт// Український медичний часопис. - № 5 (133), T. 1. - 2019. - С. 12-14.

12. Энергетическая система человека как часть природного энергообмена: значение для фундаментальной науки и медицины (часть II) / М. М. Потяженко, А. В. Невойт // Український медичний часопис. - 2019. — № 6 (134), Т. 1. - С. 41-44.

13. Фотоэлектронная (ГРВ) эмиссия как отражение микроциркуляторных флуктуаций / О. В. Сорокин, А. И. Хаданов, К. Г. Коротков, В. Ю. Куликов // Медицинские науки. - 2010. — № 1. — Режим доступу: https://cyberleninka.ru/article/n/ fotoelektronnaya — grv—emissiya_-kak—otrazheniemikrotsirkulyatornyh-fluktuatsiy. 
14. Predictive, Personalized, Preventive and Participatory (4P) Medicine Applied to Telemedicine and eHealth in the Literature / Alonso S. G., de la Torre Díez I., Zapiraín B. G. // J. Med Syst. — 2019. — № 43. — P. 140.

15. Avijgan M. Can the Primo Vascular System (Bong Han Duct System) be a Basic Concept for Qi Production? / M. Aviigan // International Journal of Integrative Medicine. - 2013. - Vol. 1. - P. 1-10.

16. The use of bioelectrography in the comparison between women with breast cancer, healthy sedentary women and women who run / K. H. Buck, C. Novelli, F. T. Costa et al. // Center for Advanced Research on Quality of Life. - 2016. - Vol. 8 (2). — P. 9-11.

17. Ultra-weak photon emission from biological samples: definition, mechanisms, properties, detection and applications / M. Cifra, P. Pospíšil // Journal of Photochemistry and Photobiology B: Biology. — 2014. — Vol. 139. - P. 2-10.

18. Device for measuring electromagnetic field intensity. Available from: https://www.iumab.org/device-formeasuring-electromagnetic-field-intensity/.

19. Risk factors for incident hospitalized heart failure with preserved versus reduced ejection fraction in a multiracial cohort of postmenopausal women / C. B. Eaton, M. Pettinger, J. Rossouw et al. // Circ. Heart Fail. - 2016. - Vol. 9. — P. e002883.

20. A nanoelectronics-blood-based diagnostic biomarker for myalgic encephalomyelitis/chronic fatigue syndrome (ME/CFS) / R. Esfandyarpour, A. Kashi, M. NematGorgani et al. // PNAS. - 2019. — Vol. 116 (21). P. 10250-10257.

21. Electrophotonic imaging technology in the diagnosis of autonomic nervous system in patients with arterial hypertension / K. G. Korotkov, I. E. Korobka, E. G. Yakovleva, S. S. Belonosov, T. V. Kolesnichenko // J. Appl Biotechnol Bioeng. — 2018. — Vol. 5 (1). — P. 20-25.

22. Randomized study of the effects of quantum resonance technology application on human psychophiological parameters / K. G. Korotkov, O. A. Churganov, E. A. Gavrilova // J. of Applied Biotechnology and Bioengineering. - 2020. — Vol. 7 (3). — P. 95-98.

23. Effect of Integrated Yoga Program on Energy Outcomes as a Measure of Preventive Health Care in Healthy People / K. K. Kushwah, H. R. Nagendra, T. M. Srinivasan // Central European Journal of Sport Sciences and Medicine. — 2015. — Vol. 12 (4). — P. 61-71.

24. Kwang-Sup Soh. Bonghan Circulatory System as an Extension of Acupuncture Meridians / Kwang-Sup Soh // Journal of Acupuncture and Meridian Studies. — 2009. — Vol. 2 (2). - P. 93-106.

25. Kwang-Sup Soh. The Primo Vascular System. Its role in cancer and regeneration / Kwang-Sup Soh, Kyung A. Kang, David K. Harrison. - Springer, 2012. - 336 p.

26. Update on obesity and obesity paradox in heart failure / Lavie C. J., Sharma A., Alpert M. A. et al. // Prog. Cardiovasc. Dis. — 2016. — Vol. 58 (4). — P. 393-400.
27. Evaluation of the human bioelectromagnetic field in medicine: the development of methodology and prospects are at the present scientific stage / O. P. Mintser, M. M. Potiazhenko, G. V. Nevoit // Wiadomości Lekarskie - 2019. — Vol. 5, II. — P. 1117-1121.

28. Obesity and subtypes of incident cardiovascular disease / C. E. Ndumele, K. Matsushita, M. Lazo et al. // J. Am. Heart Assoc. — 2016. — Vol. 5. — P. e003921.

29. The obesity paradox and heart failure: a systematic review of a decade of evidence / E. A. Oga, O. R. Eseyin // J. Obes. - 2016. — P. 9040248.

30. Ultraweak Photon Emission in the Brain / V. Salari, H. Valian, H. Bassereh, I. Bókkon, A. Barkhordari // J. Integr. Neurosci. — 2015. — Vol. 14. — P. 419-429.

31. History of Bioelectrical Study and the Electrophysiology of the Primo Vascular System / S. H. Park, E. H. Kim, H. J. Chang et al. // Evidence-based Complementary and Alternative Medicine. - 2013. - 14 p.

32. Non-Chemical and Non-Contact Cell-to-Cell Communication: A Short Review / F. Scholkmann, D. Fels, M. Cifra // Am. J. Trans. Res. — 2013. — Vol. 5. - P. 586-593.

33. Electrophotonic Imaging Based Analysis of Diabetes / K. K. Shiva, H. R. Nagendra, T. M. Srinivasan et al. // International Journal Alternative and Complementary Medicine. - 2016. — Vol. 4 (5). - P. 134-137.

34. The Primo Vascular system as a New Anatomic System / M. Stefanov, M. Potroz, J. Kim et al. // Journal of Acupuncture and Meridian Studies. — 2013. — Vol. 6 (6). - P. 331-338.

35. Comparative study of Influence of Yajña and Yogasana on stress level as Measured by Electron Photonic Imaging (EPI) Technique / S. Sushrutha, M. Hegde, H. R. Nagendra et al. // International Journal Science and Research. - 2014. - Vol. 8. - P. 1402-1406.

36. Direct Detection of a Single Photon by Humans / Tinsley J. N., Molodtsov M. I., Prevedel R. et al. // Nat. Commun. - 2016. — Vol. 7. - P. 12-17.

37. Identifying Patients with Colon Neoplasias with Gas Discharge Visualization Technique / T. G. Yakovleva, O. A. Buntseva, S. S. Belonosov et al. // J. Alternative and Complementary Medicine. — 2015. — Vol. 21. P. 720-724.

\section{References.}

1. Bojko, V. V., Krasnogolovec. M. A. (2003). Kvantovobiologicheskaya teoriya [Quantum Biological Theory]. Harkov: Fakt, 967. [In Ukrainian].

2. Bojko, V. V., Sokol, E. I., Zamyatin, P. H. (2016). Pezobiosintez: predposylki, gipotezy, fakty [Piezobiosynthesis: premises, hypotheses, facts]. Harkov: Hark. politehn. in-t, 4t. [In Ukrainian].

3. Vojcehovska, K. V., Voronkov, L. G. (2019). Prognostichne znachennya vtrati masi tila ta pokaznikiv antropometriyi u paciyentiv iz hronichnoyu sercevoyu nedostatnistyu ta znizhenoyu frakciyeyu vikidu livogo 
shlunochka [Prognostic value of weight loss and anthropometry in patients with chronic heart failure and reduced left ventricular ejection fraction]. Ukrayinskij medichnij chasopis. (Ukrainian Medical Chronicle), 5 (2), 31-4. [In Ukrainian].

4. Davydov, A. S. (1984). Solitoni v molekulyarnih sistemah [Solitons in molecular systems]. Kyiv: Naukova dumka, 288. [In Russian].

5. Doroshenko, L.Yu. (2020). Variabelnost serdechnogo ritma: zabytoe nasledie kosmicheskoj mediciny SSSR [Heart Rate Variability: The Forgotten Legacy of USSR Space Medicine]. Moya avtomatika i ne tolko...(My automation and not only ...). Retrieved from: http:// vdd-pro.ru/ru/2020/01/hrv-analysis-forgotten-heritage/. [In Russian].

6. Kovalenko, O. Ye., Chizhikova, M. Ye. (2017). Suchasni poglyadi na substrat ta mehanizmi diyi akupunkturi [Modern views on the substrate and mechanisms of action of acupuncture]. Mizhnarodnij nevrologichnij zhurnal. (International Journal of Neurology), 6, 120-6. [In Ukrainian].

7. Kotova, O. V., Akarachkova, E. S. (2016). Astenicheskij sindrom v praktike nevrologa i semejnogo vracha [Asthenic syndrome in the practice of a neurologist and family doctor]. Russkij medicinskij zhurnal. (Russian medical journal), 13, 824-9. [In Russian].

8. Mintcer, O. P., Zaliskij, V. M. (2019). Sistemna biomedicina (u dvoh tomah) T. 1. Konceptualizaciya [Systemic biomedicine (in two volumes) Vol. 1. Conceptualization]. Kyiv: Interservis, 552. [In Ukrainian].

9. Pizova, N. V. (2015). Klinika, diagnostika i lechenie sindroma hronicheskoj ustalosti [Clinic, diagnosis and treatment of chronic fatigue syndrome]. Medicinskij sovet. (Medical advice), 2, 20-4. [In Russian].

10. Potyazhenko, M. M., Nevojt, A. V. (2019). Energeticheskaya sistema cheloveka v svete sovremennyh fiziko-biologicheskih znanij, koncepcij, gipotez [The human energy system in the light of modern physical and biological knowledge, concepts, hypotheses]. Ukrayinskij medichnij chasopis. (Ukrainian Medical Chronicle), 4 (132), 2, 24-9. [In Russian].

11. Potyazhenko, M. M., Nevojt, A. V. (2019). Energeticheskaya sistema cheloveka kak chast prirodnogo energoobmena: znachenie dlya fundamentalnoj nauki i mediciny (chast I) [The human energy system as part of natural energy exchange: importance for fundamental science and medicine (part I)]. Ukrayinskij medichnij chasopis. (Ukrainian Medical Chronicle), 5 (133), 1, 12-4. [In Russian].

12. Potyazhenko, M. M., Nevojt, A. V. (2019). Energeticheskaya sistema cheloveka kak chast prirodnogo energoobmena: znachenie dlya fundamentalnoj nauki i mediciny (chast II) [The human energy system as part of natural energy exchange: importance for fundamental science and medicine (part II)]. Ukrayinskij medichnij chasopis. (Ukrainian Medical Chronicle), 6 (134), 1, 41-4. [In Russian].

13. Sorokin, O. V., Hadanov, A. I., Korotkov, K. G., Kulikov, V. Yu. (2010). Fotoelektronnaya (GRV) emissiya kak otrazhenie mikrocirkulyatornyh fluktuacij [Photoelectron (GDV) emission as a reflection of microcirculatory fluctuations]. Medicinskie nauki. (Medical sciences), 1, Retrieved from: https:/cyberleninka.ru/article/n/ fotoelektronnaya-grv-emissiya-kak-otrazheniemikrotsirkulyatornyh-fluktuatsiy.

14. Alonso, S. G, de la Torre Díez, I., Zapiraín, B. G. (2019). Predictive, Personalized, Preventive and Participatory (4P) Medicine Applied to Telemedicine and eHealth in the Literature. J Med Syst., 43, 140. Retrieved from: https://doi.org/10.1007/s10916-019-1279-4.

15. Avijgan, M. (2013). Can the Primo Vascular System (Bong Han Duct System) be a Basic Concept for Qi Production? International Journal of Integrative Medicine, 1, 1-10.

16. Buck, K. H., Novelli, C., Costa, F. T. et al. (2016). The use of bioelectrography in the comparison between women with breast cancer, healthy sedentary women and women who run. Center for Advanced Research on Quality of Life., 8 (2), 9-11.

17. Cifra, M., Pospíšil, P. (2014). Ultra-weak photon emission from biological samples: definition, mechanisms, properties, detection and applications. Journal of Photochemistry and Photobiology. 139, 2-10. B: Biology. Retrieved from: https://doi.org/10.1016/j. jphotobiol.2014.02.009

18. Device for measuring electromagnetic field intensity. Retrieved from: https://www.iumab.org/device-formeasuring-electromagnetic-field-intensity/.

19. Eaton, C. B., Pettinger, M., Rossouw, J. et al. (2016). Risk factors for incident hospitalized heart failure with preserved versus reduced ejection fraction in a multiracial cohort of postmenopausal women. Circ. Heart Fail., 9, 002883.

20. Esfandyarpour, R., Kashi, A., Nemat-Gorgani, M. et al. (2019). A nanoelectronics-blood-based diagnostic biomarker for myalgic encephalomyelitis/chronic fatigue syndrome (ME/CFS). PNAS, 116 (21), 1025057.

21. Korotkov, K. G., Korobka, I. E., Yakovleva, E. G., Belonosov, S. S., Kolesnichenko, T. V. (2018). Electrophotonic imaging technology in the diagnosis of autonomic nervous system in patients with arterial hypertension. J. Appl Biotechnol Bioeng., 5 (1), 20-5.

22. Korotkov, K. G., Churganov, O. A., Gavrilova, E. A. (2020). Randomized study of the effects of quantum resonance technology application on human psychophiological parameters. J. of Applied Biotechnology and Bioengineering, 7 (3), 95-8.

23. Kushwah, K. K., Nagendra, H. R., Srinivasan, T. M. (2015). Effect of Integrated Yoga Program on Energy Outcomes as a Measure of Preventive Health Care in 
Healthy People. Central European Journal of Sport Sciences and Medicine, 12 (4), 61-71.

24. Kwang-Sup Soh, (2009). Bonghan Circulatory System as an Extension of Acupuncture Meridians. Journal of Acupuncture and Meridian Studies, 2(2), 93-106. Retrieved from: https://doi.org/10.1016/S20052901(09)60041-8.

25. Soh, K.-S., Kang, K. A., Harrison, D. K. (2012). The Primo Vascular System. Its role in cancer and regeneration. Springer, 336.

26. Lavie C. J., Sharma A., Alpert M. A. et al. (2016). Update on obesity and obesity paradox in heart failure. Prog. Cardiovasc. Dis., 58 (4), 393-400.

27. Mintser, O. P., Potiazhenko, M. M., Nevoit, G. V. (2019). Evaluation of the human bioelectromagnetic field in medicine: the development of methodology and prospects are at the present scientific stage. Wiadomości Lekarskie, 5 (II), 1117-21.

28. Ndumele, C. E., Matsushita, K., Lazo, M. (2016). Obesity and subtypes of incident cardiovascular disease. J. Am. Heart Assoc., 5, 003921.

29. Oga, E. A., Eseyin, O. R. (2016). The obesity paradox and heart failure: a systematic review of a decade of evidence. J. Obes., 9040248.

30. Salari, V., Valian, H., Bassereh, H., Bókkon I., Barkhordari, A. (2015). Ultraweak Photon Emission in the Brain. J. Integr. Neurosci., 14, 419-29.
31. Park, S. H., Kim, E. H., Chang, H. J. et al. (2013). History of Bioelectrical Study and the Electrophysiology of the Primo Vascular System. Evidence-based Complementary and Alternative Medicine, 14. Retrieved from: https://doi.org/10.1155/2013/486823.

32. Scholkmann, F., Fels, D., Cifra, M. (2013). Non-Chemical and Non-Contact Cell-to-Cell Communication: A Short Review, Am. J. Trans. Res., 5, 586-93.

33. Shiva, K. K., Nagendra, H. R., Srinivasan, T. M., Marimuthu, P. (2016). Electrophotonic Imaging Based Analysis of Diabetes. International Journal Alternative and Complementary Medicine, 4 (5), 134-7.

34. Stefanov, M., Potroz, M., Kim, J., (2013). The Primo Vascular system as a New Anatomic System. Journal of Acupuncture and Meridian Studies, 6 (6), 331-8.

35. Sushrutha, S. Hegde, M., Nagendra, H.R., Srinivasan T. M. (2014). Comparative study of Influence of Yajña and Yogasana on stress level as Measured by Electron Photonic Imaging (EPI) Technique. International Journal Science and Research., 8, 1402-06.

36. Tinsley, J. N., Molodtsov, M. I., Prevedel, R. et al. (2016). Direct Detection of a Single Photon by Humans. Nat. Commun., 7, 12-7.

37. Yakovleva, E. G., Buntseva, O. A., Belonosov, S. S. et al. (2015). Identifying Patients with Colon Neoplasias with Gas Discharge Visualization Technique. J. Alternative and Complementary Medicine., 21, 720-4. 